\title{
Publisher Correction: Oil exposure disrupts early life-history stages of coral reef fishes via behavioural impairments
}

Jacob L. Johansen, Bridie J. M. Allan, Jodie L. Rummer and Andrew J. Esbaugh

Nature Ecology \& Evolution 1, 1146-1152 (2017); published online 17 July 2017; corrected online 2 August 2017

In the version of this Article originally published, a statistic relating to the northern Great Barrier Reef was attributed to the Great Barrier Reef as a whole. The sentence should have read 'In 2016 alone, more than 35\% of corals on the northern Great Barrier Reef are estimated to have died following the worst bleaching event ever recorded'. This has been corrected in all versions of the Article. 